\title{
sciendo
}

ISSN: $1231-4005$

e-ISSN: 2354-0133

DOI: $10.2478 /$ kones-2019-0110

\section{INVESTIGATION OF THERMAL PROPERTIES OF NOVEL PHASE CHANGE MATERIAL MIXTURES (OCTADECANE-DIAMOND) WITH LASER FLASH ANALYSIS}

\author{
Mateusz Sierakowski, Wojciech Godlewski \\ Roman Domański, Jakub Kapuściński \\ Łukasiewicz Research Network - Institute of Aviation \\ Krakowska Av. 110/114, 02-256 Warsaw, Poland \\ e-mail:m.sierako@gmail.com,wojtgod@wp.pl \\ roman.domanski@ilot.edu.pl,jakub.kapuscinski@ilot.edu.pl \\ Tomasz Wiśniewski, Michal Kubiś \\ Warsaw University of Technology \\ Nowowiejska Street 21/25, 00-665 Warsaw, Poland \\ e-mail:tomasz.wisniewski@itc.pw.edu.pl,michal.kubis@itc.pw.edu.pl
}

\begin{abstract}
Phase change materials (PCMs) are widely used in numerous engineering fields because of their good heat storage properties and high latent heat of fusion. However, a big group of them has low thermal conductivity and diffusivity, which poses a problem when it comes to effective and relatively fast heat transfer and accumulation. Therefore, their use is limited to systems that do not need to be heated or cooled rapidly. That is why they are used as thermal energy storage systems in both large scale in power plants and smaller scale in residential facilities. Although, if PCMs are meant to play an important role in electronics cooling, heat dissipation, or temperature stabilization in places where the access to cooling water is limited, such as electric automotive industry or hybrid aviation, a number of modifications and improvements needs to be introduced. Investigation whether additional materials of better thermal properties will affect the thermal properties of PCM is therefore of a big interest. An example of such material is diamond powder, which is a popular additive used in abradants. Its thermal diffusivity and conductivity is significantly higher than for a pure PCM. The article presents the results of an analysis of the effect of diamond powder on thermal conductivity and diffusivity of phase change materials in the case of octadecane.
\end{abstract}

Keywords: phase change materials, PCM, diamond powder, thermal conductivity, thermal diffusivity

\section{Introduction}

Among numerous types of energy storage technologies is thermal energy storage (TES). It is based on accumulating heat or cold that is later used for different applications [1]. Phase change materials are commonly used in this field [2]. They are similar to other materials that are sensible heat stores in that their temperature increases while absorbing heat, but when they reach the melting point, they absorb a significant amount of heat at a constant temperature [3]. This leads to a substantial delay in terms of achieving a specific value of temperature of the system or component, because the melting PCM consumes the excess of energy produced by the system. There are PCMs which, compared to conventional latent heat stores (water, soil, etc.), are able to store up to ten times more heat per unit of volume. There is a need to introduce more effective heat sinks in electronics and mechanical systems' cooling [4], which are more responsive and able to transport and accumulate heat at higher rates. Therefore, research is carried out on new composites that link the strongest sides of its constituents. This article presents the results of an investigation of the impact of diamond powder on thermal diffusivity and conductivity of octadecane, which is a paraffin wax belonging to the group of phase change materials. 
This substance was chosen because of its very good thermal properties and its relative availability. Diamond particle additive has not been thoroughly investigated in mixtures with PCMs so far. Therefore, more research into this subject was done. It was expected that a faster thermal response and substantial thermal conductivity enhancement of new composites would occur compared to the values describing a pure PCM [5]. The impact of three different diamond grain sizes and different mass ratios of diamond powder in the mixture is shown. Additionally, a problem of particle sedimentation and preservation of mixture consistency are described. Final composites were enriched with amorphous silicon dioxide to overcome this problem. Therefore, the influence of this material itself on the thermal properties of the tested specimens in newly formed composites was also examined.

\section{Description of the experiment and used materials}

\subsection{Octadecane}

Octadecane is an alkane with a straight chain. It has 18 atoms of carbon. Its molecular formula is $\mathrm{C} 18 \mathrm{H} 38$. Most important parameters of octadecane are presented in Tab. 1 [6-8].

Tab. 1. Basic physical properties of octadecane

\begin{tabular}{|l|c|}
\hline Molecular weight & $254.502 \mathrm{~g} / \mathrm{mol}$ \\
\hline Boiling point & $316^{\circ} \mathrm{C}$ \\
\hline Melting point & $28^{\circ} \mathrm{C}$ \\
\hline Flash point & $166^{\circ} \mathrm{C}$ \\
\hline Density & $0.777 \mathrm{~g} / \mathrm{cm}^{2}$ at $28^{\circ} \mathrm{C}$ \\
\hline Thermal conductivity & $0.3 \mathrm{~W} / \mathrm{m} \cdot \mathrm{K}$ \\
\hline Thermal diffusivity & $0.21 \mathrm{~mm} / \mathrm{s}$ \\
\hline
\end{tabular}

\subsection{Diamond}

Depending on diamond's internal structure, its thermal conductivity can range from 900 to over $3500 \mathrm{~W} / \mathrm{m} \cdot \mathrm{K}[9,10]$. A high value of thermal diffusivity, which combines thermal conductivity, material's density and thermal capacity, can reach $1100 \mathrm{~mm}^{2} / \mathrm{s}$. In this research, diamond was used in a form of yellow powder of three different grain sizes: 50, 150 and 250 micrometres.

\subsection{Laser flash analysis}

Laser flash analysis (LFA) is a method used to measure thermal diffusivity of materials by providing them with a short light or laser pulse. It hits one surface of the sample whose thickness is measured before the experiment. This value is usually less than $1 \mathrm{~mm}$. The heat from the laser beam then is absorbed by the face of the specimen and is transported through the investigated material.

At the second face side, high-speed infrared sensors record the changes of temperature with respect to time [11]. With LFA, one can also indirectly determine thermal conductivity, knowing specific heat and density of the measured material. A simplified mechanism of this method and measurement apparatus is presented in Fig. 1.

\subsection{Preparation of the samples and mixing}

The amount of diamond powder available for the experiment made it very hard to use ultrasonic homogenizer for mixing because of relatively big dimensions of the mixing equipment 
with respect to very small specimens. Thus, it was decided to mix the samples manually. To eliminate stratification phenomenon it was decided to add amorphous silicon dioxide to the mixtures. The content of this material was determined experimentally, with constant PCM to diamond powder ratio, which equalled $1: 1$.

a)

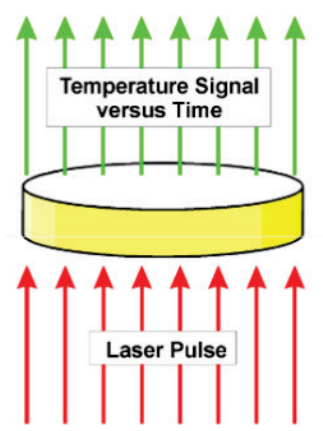

b)

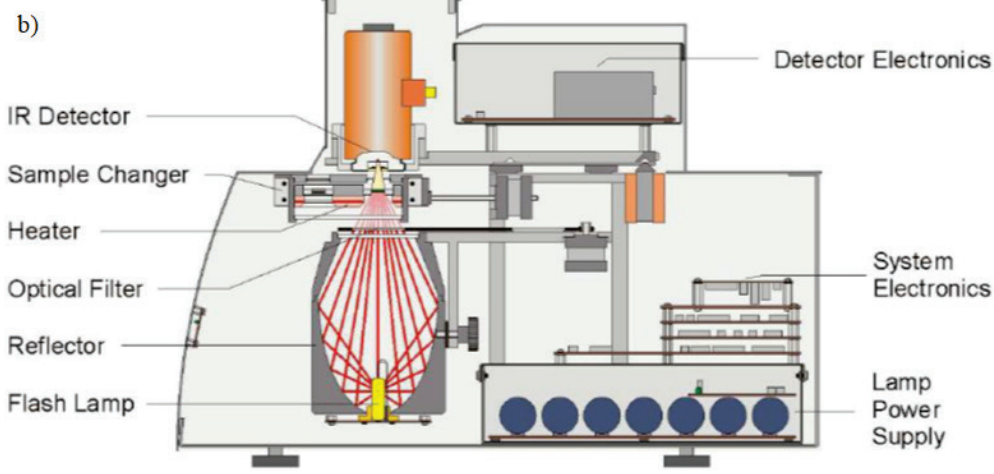

Fig. 1. General principle of operation of LFA (a) and main parts of the apparatus (b) [12]

The specimens have undergone melting - solidification cycle testing. The number of cycles ranged from 20 to 40 . The starting temperature was set to be $15^{\circ} \mathrm{C}$ to make sure the mixture was in solid state. The maximum temperature was $35^{\circ} \mathrm{C}$.

After several attempts it was determined that at a 4\%-mass fraction of amorphous silicon dioxide caused the mixture to have a form of a paste. Therefore, this value was set as the most optimal one. Increased content of this substance would lead to decreased heat capacity of the mixture and bigger probability that it is inhomogeneous.

Finally, the specimen was put onto the sample holder and closed with the lid.

\section{Thermal diffusivity measurement}

Firstly, thermal diffusivity of pure octadecane was examined. It served as a reference in comparison of new mixtures with the original pure form. Averaged values from the experiment are shown in the Fig. 2 a.

Thermal diffusivity of pure octadecane is higher in a solidified form than as a liquid. It has a beneficial effect on the performance of such a substance as a cooling material because the solid form (before the phase change) has a better temperature response time.

Before the principal measurements were conducted, there was a need to evaluate the influence of amorphous silicon dioxide on thermal diffusivity of the mixture of octadecane and amorphous silicon dioxide in different mass ratios. The results are presented in Fig. $2 b$.
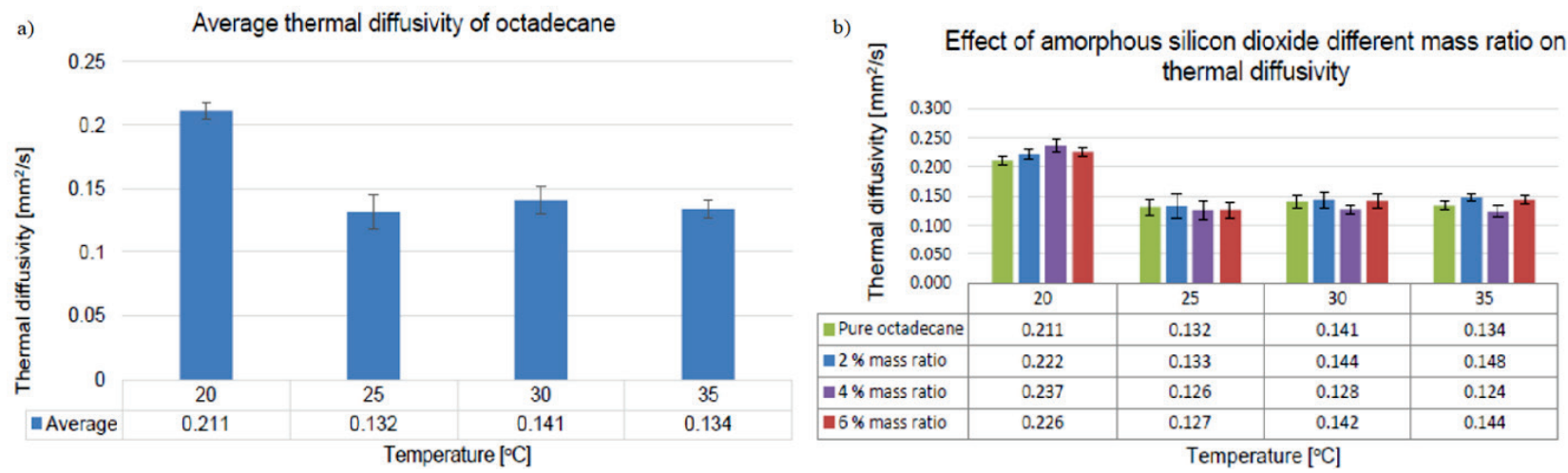

Fig. 2. Experimental data on thermal diffusivity of octadecane for 4 temperature levels (a) and thermal diffusivity of the mixture of the PCM with amorphous silicon dioxide for 4 temperature levels (b) 
It can be observed that amorphous silicon dioxide has very little influence on thermal diffusivity of the newly formed mixture. Considering standard deviation, an assumption can be made that the changes of thermal diffusivity in the subsequent experiments may be attributed only to the presence of diamond particles. Therefore, a big dose of attention should be paid to the effect of different mass percentages and sizes of diamond powder in the mixtures.

The core of the research was concentrated on the influence of diamond powder on overall thermal diffusivity of the PCM mixtures with different grain sizes and mass percentages of the powder. A complex analysis was performed for the grain sizes of 50 and 250 micrometres. This could give an information on the tendency of the material to diffuse heat with respect to increasing the grain size.

\subsection{The effect of diamond powder of 50 and 250 -micrometre grain size}

The results are presented in the Fig. 3.
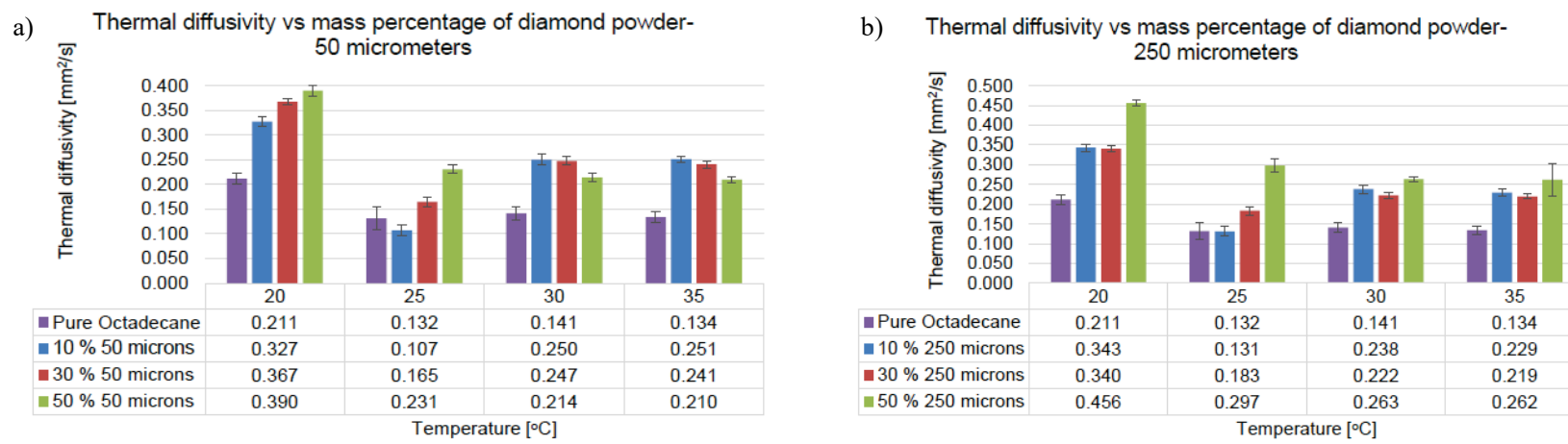

Fig. 3. Thermal diffusivity of PCM - diamond composite with 50 micrometre (a) and 250-micrometre (b) diamond grain size with three different mass ratios for four temperature levels

\section{0-micrometre grain size}

It is visible in the Fig. 3a that diamond powder significantly increases thermal diffusivity of the substance. The value increases in the solid state $\left(20^{\circ} \mathrm{C}\right)$ as there is a bigger mass fraction of diamond powder, but it should be noted that between $10 \%$ and $50 \%$ mass fraction the difference is not that big. In the liquid state thermal diffusivity is similar in all of the cases $\left(30^{\circ} \mathrm{C}\right.$ and $\left.35^{\circ} \mathrm{C}\right)$. However, the most important observation in this case is for the solid state because primarily this phase is responsible for initial heat removal. A noticeable jump in thermal diffusivity is marked even for $10 \%$ mass fraction. This is a good information because such a configuration is more economically viable and maintains good level of heat capacity of the composite.

\section{0-micrometre grain size}

Figure $3 \mathrm{~b}$ suggests that in the solid state $50 \%$ mass fraction of the diamond powder behaves best because it has the highest thermal diffusivity. However, other mass fractions have the same impact on overall thermal diffusivity, which is an interesting result. It should be noted that the samples have the thickness of 600-700 micrometres and with such a low value and relatively big grain sizes, the homogeneity of the specimen could be not invariant across it despite homogeneous mixing beforehand because there could occur diamond grain concentrations and holes that lead to incorrect result. In the liquid state the case is very similar to the one with 50 micrometres - thermal diffusivity is lower than in solid state and is very similar for every mass percentage of diamond powder. It can be observed that for this granulation and 50\% mass percentage of diamond powder overall thermal diffusivity is the biggest out of the whole group of newly formed mixtures. The reason for this might be that with big grain size and concentration of diamond, powder connections are more ubiquitous and therefore the conduction and diffusion of heat is more intense. With 
smaller particles like 150 and 50 micrometres, these connections are less likely to be built in a bigger scale along the mixture.

Due to time limits and clear conclusion from last measurements, that thermal diffusivity of the mixture increases with the size of diamond grains only $50 \%$ mass fraction with the grain size of 150 micrometres was investigated (Fig. 4a).

The results proved that there is a rising tendency of thermal diffusivity with rising grain size, because the enhancement level was located in the middle range between 50 and 250-micrometre grain size. From the taken measurements of thermal diffusivity of the investigated composites, it can be concluded that thermal diffusivity rises when the mass ratio of diamond powder increases. However, for the case of solid case, which was crucial in this experiment, the differences between enhancement level with $10 \%$ and $50 \%$ mass ratio of diamond powder were not so big for the mixture with 50 micrometres (Fig. 4b).
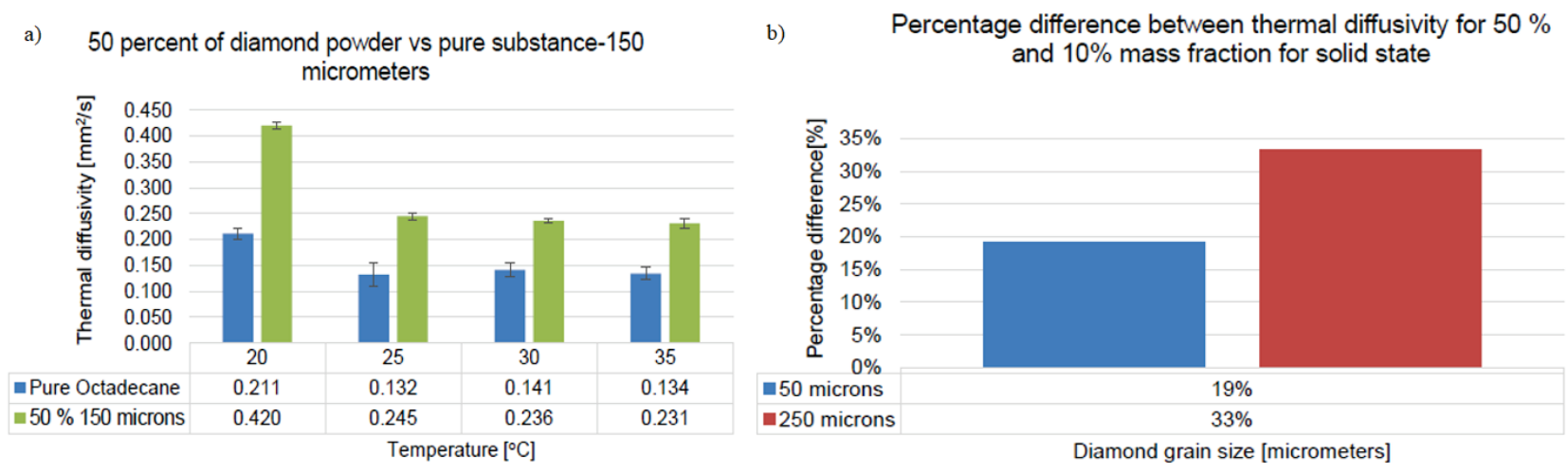

Fig. 4. Thermal diffusivity of PCM - diamond composite with 150 micrometre diamond grain size with 50\% mass ratio for four temperature levels (a) and the difference of thermal diffusivity between $P C M$ - diamond composites with grains of the diameter of 50 and 250 micrometres (b)

The mixtures with the grain size of 50 micrometres were the most resistant to sedimentation after several melting-solidification cycles. Thus, they should be examined thoroughly because with $185 \%$ increase for $50 \%$ mass ratio and good cycling properties this configuration is one of the best ones obtained in this experiment. Another good result was obtained from the mixture with 250 micrometres and 50\% mass ratio because improved thermal diffusivity exceeded $200 \%$. In addition, there is a problem with sedimentation; it should be taken into account when for this substance it comes to the choice of application.

\section{Thermal conductivity}

After the experiments, specific heat capacity and thermal diffusivity have been found for specific conditions and composition of the mixtures. Density was also calculated. It should be noted that specific heat capacity for the temperature of $20^{\circ} \mathrm{C}$ was calculated, not found from differential scanning calorimetry (DSC) results. This is because of the fact that for good determination of this value the heating rate in DSC must be changed to lower value, which would lead to frost production on certain components of DSC apparatus and further to incorrect measurements and their bad quality.

In this section, the results for the temperature of $25^{\circ} \mathrm{C}$ are not shown due to unstable behaviour of the material and difficulty in determination of accurate and correct readings of specific heat capacity and thermal diffusivity. Due to the time limits and experience gained in previous experiments the grain size of 150 micrometres was not used here. 50 and 250-micrometre sizes were predicted to show the desired outcome. The Authors predicted that thermal conductivity would rise in a manner similar to thermal diffusivity. This prediction came out to be true. The results can be seen in the Fig. 5 . 
a)

10 percent of diamond powder of different granulation in different temperatures

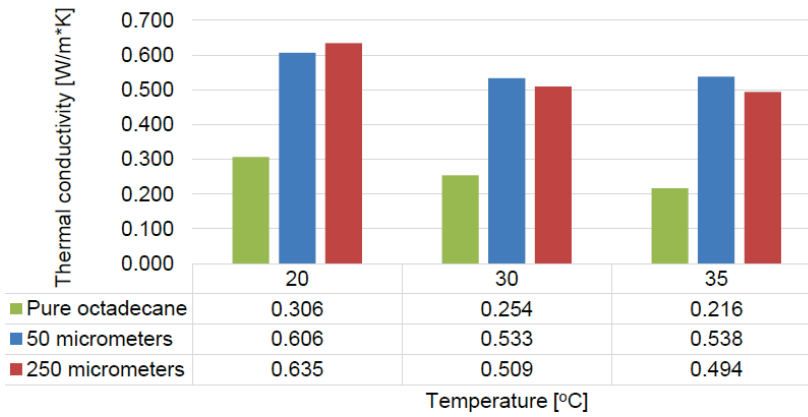

b)

30 percent of diamond powder of different granulation in different temperatures

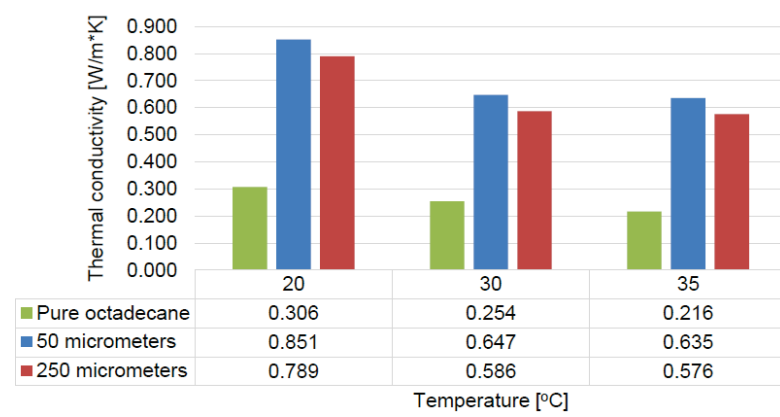

Fig. 5. Thermal conductivity of PCM - diamond composite with 50 micrometre and 250 micrometre diamond grain size with $10 \%$ (a) and $30 \%$ (b) mass ratios for three temperature levels

It can be seen in the Fig. 5a that thermal conductivity approximately doubled its value after addition of diamond powder. In this case, the impact of grain size was almost negligible. This is a good result because small addition of the material leads to relatively big increase of the examined value. Thermal conductivity in the liquid state tends to be approximately constant.

Another analysis concerned 30\% mass ratio for the case of different diamond grain sizes. The results are presented in the Fig. 5b. The most important conclusion from the above-shown graph is that thermal conductivity rises with increased mass ratio of diamond powder. The enhancement level is exceeding $200 \%$. The results for the temperature of $20^{\circ} \mathrm{C}$ show that again the grain size in this mass fraction does not have a big influence on thermal conductivity. Therefore, it is better to use smaller grains because as the experiments showed, smaller granulation deals better in meltingsolidification cycles. In the liquid state, thermal conductivity changes are very small.

The last comparison shows the behaviour of the mixture with diamond powder to PCM mass ratio equal to $50 \%$ (Fig. $6 \mathrm{a}$ ).
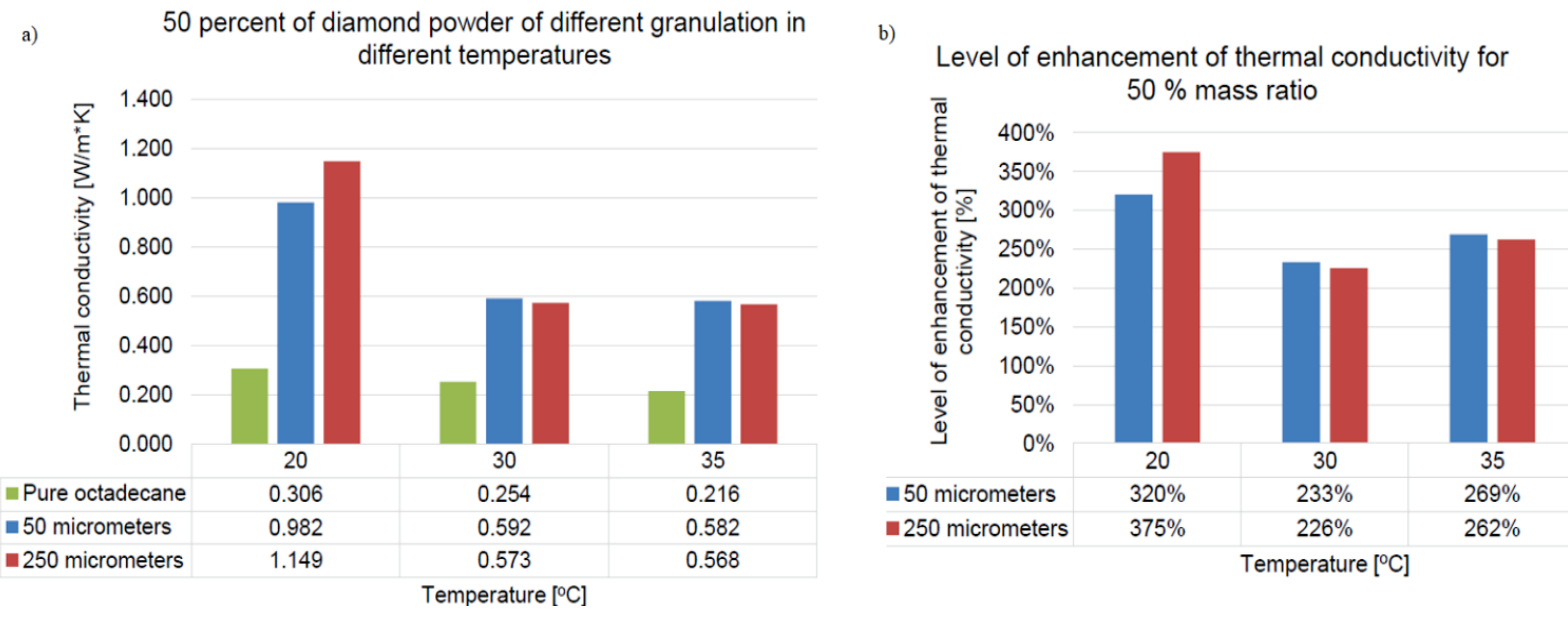

Fig. 6. Thermal conductivity of PCM - diamond composite for the case of 50\% mass ratio for three temperature levels, grain sizes: 250 and 50 micrometres (a) and level of enhancement of thermal conductivity for the case of 50\% mass ratio for 250 and 50-micrometre grain size (b)

In this case, mass fraction of diamond powder thermal conductivity in the liquid state is very similar as in the previous cases. However, there is a rising tendency of this value in the solid state. This might be because with this content of diamond filler there is a bigger probability that big grains touch together and create a structure across the material that conducts heat faster compared to the material with smaller grains, where such structures due to bigger gaps between neighbouring particles are not formed. Finally, in the case of bigger grains of diamond powder, there are less particles compared to the same unit mass of smaller granulation and therefore the interfacial 
thermal resistance is less because there are less PCM-diamond and amorphous silicon dioxidediamond boundaries [13].

Summing up the advance in thermal conductivity in the investigated materials, the best results are noted for $50 \%$ mass fraction of diamond powder. It can be seen in the Fig. 6 b.

The chart delivers very optimistic information that thermal conductivity for $50 \%$ mass ratio of diamond powder is over 350\% bigger than for the pure PCM. Even though this value is the biggest for the largest investigated grain size, the performance of the composite with the smallest grain size is also very good-it is also over three times better than the performance of the pure substance. It is important to mention that the mixture with 50-micrometre diamond grain size has bigger resistance to cycling and therefore is a more durable material, whose thermal conductivity enhancement level is only 55 percentage points smaller than the thermal conductivity of the composite with 250-micrometre grain size.

\section{Conclusions}

The predictions about increased thermal diffusivity and conductivity of the PCM-diamond composites came out to be true. With increasing size of the diamond grains in the mixture, those parameters increased as well. This is because bigger grains have bigger potential to touch themselves and create a heat conducting structure across the material. Increasing the mass percentage of diamond in the mixture had also a positive effect on thermal conductivity and diffusivity. A good result was recorded even for $10 \%$ mass fraction and the difference between this and $30 \%$ mass fraction of diamond powder was relatively small. Therefore, for some applications it can be a good idea not to add too much of the filler, e.g. diamond powder, to save good value of specific heat and latent heat.

Because it was observed that despite an increased mass fraction of amorphous silicon dioxide especially for the bigger granulation the first signs of stratification start appearing after approximately 20 cycles of melting and solidification, an application of such materials must be chosen appropriately.

One of the applications of the tested mixtures could be a thermal fuse in electronics, when other cooling systems do not manage to absorb required amount of heat. If the temperature reaches a certain level, the PCM composite with diamond grains starts to melt, consuming heat that is produced in electronic systems. Diamond powder helps to transport heat in a faster way from the system to the container with PCM composite.

The time limits of the experiment did not allow for more thorough research into determination of more stable and homogeneous mixtures. For each case of different mass ratios, an optimal content of amorphous silicon dioxide should be found.

It should be noted that this material is not the only one that has such properties. Further research might concentrate on the behaviour of PCM-diamond composites with a substance that acts better as a viscosity-increasing agent.

Finally, a very interesting solution is the usage of PCMs with conductive foam with additives having very good thermal properties [14]. In this case, the foam keeps its structure in the container, where the pores are filled with the PCM. Here the problem of sedimentation is skipped because the conductive structure is rigid and does not decompose because of temperature variations and PCM phase changes. However, this solution can be substantially more expensive than the solution presented in this article.

\section{References}

[1] Domański, R., Magazynowanie energii cieplnej, 1990.

[2] Banaszek, J., Domański, R., Rebow, M., El-Sagier, F., Experimental study of solid-liquid phase change in a spiral thermal energy storage unit, Applied Thermal Engineering, Vol. 19, Iss. 12, pp. 1253-1277, 1999. 
[3] Mehling, H., Cabeza, L. F., Heat and cold storage with PCM, 2008.

[4] Jaworski, M., Thermal performance of heat spreader for electronics cooling with incorporated phase change material, Applied Thermal Engineering, October 2011.

[5] Sharma, R. K., Ganesan, P.,Tyagi, V. V., Metselaar, H. S. C., Sandaran, S. C., Developments in organic solid-liquid phase change materials and their applications in thermal energy storage, Energy Conversion and Management, Vol. 95, pp. 193-228, 2015.

[6] Haynes, W. M., CRC Handbook of Chemistry and Physics, CRC Press, 2015.

[7] Reaxys by Elsevier.

[8] Sigma-Aldrich, Safety Data Sheet for Octadecane, Version 5.4

[9] Anthony, T. R., Banholzer, W. F., Fleischer, J. F., Wei, et al., Thermal conductivity of isotopically enriched 12C diamond, Phys Rev B Condens Matter, 1990.

[10] Pan, L. S., Kania, D. R., Diamond: Electronic Properties and Applications, pp. 285-318, January 1995.

[11] Domański, R., Jaworski, M., Wiśniewski, T. S., Wymiana ciepła. Laboratorium dydaktyczne, 2002.

[12] NETZCH, Teaching materials and user's guide for NETZSCH 447 NanoFlash.

[13] Furmański, P., Wiśniewski, T., Banaszek, J., Thermal contact resistance and other thermal phenomena at solid-solid interface, OWPW, 2008.

[14] Zhang, L., Zhou, K., Wei, Q., Ma, L., Ye, W., Li, H., Zhou, B., Yu, Z., Lin, C.-T., Luo, J., Gan, X., Thermal conductivity enhancement of phase change materials with $3 D$ porous diamond foam for thermal energy storage, Applied Energy, Vol. 233-234, pp. 208-219, 2019.

Manuscript received 12 August 2019; approved for printing 09 December 2019 